\title{
Adjuvant Hypo Fractionated Whole Breast Radiation therapy: A Review of Literature from Bench to Clinic
}

\author{
Tareq Salah* \\ Clinical oncology and nuclear medicine Department, Faculty of medicine, Assiut university, Egypt
}

Submission: April 27, 2020; Published: May 20, 2020

*Corresponding author: Tareq Salah, Associate Professor of Clinical Oncology, Clinical oncology and nuclear medicine Department, Faculty of medicine, Assiut university, Assiut, Egypt

Abstract

Adjuvant Radiotherapy (RT) is an essential part of Breast cancer treatment with proved survival advantage . Although its radiobiological and clinical advantage becoming a standard of care practice in most centers, Hypofractionation (HF) still of a concern in certain clinical situations. Treatment Protocols are still variable across the ocean and worldwide. With the recent publications of the extreme hypofractionation we have a wide range of evidence-based protocols. This is a review of radiobiological bases and current evidence for hypofractionation in breast cancer radiation therapy starting from the basic science base up to the most recent studies.

\section{Background}

Radiotherapy (RT) is an essential part of Breast cancer adjuvant treatment with proved survival advantage. Although its radiobiological and clinical advantage becoming a standard of care practice in most centers, Hypofractionation (HF) still of a concern in certain clinical situations. Hypofractionation trials based predominantly on patients undergoing breast conservation surgery are informative for postmastectomy radiotherapy. Protocols are still variable across the ocean and worldwide. This is a review of Radiobiological bases and current evidence for hypofractionation in breast cancer radiation therapy.

\section{Introduction}

Hypofractionation trials based initially predominantly on patients undergoing breast conservation surgery are also informative for postmastectomy radiotherapy [1]. In this group of patients, most early trials used B2.2 Gy/fraction to deliver doses of 50-54 Gy to the breast [2]. Now , HF Radiation therapy with 2.5 - 3.0 Gy per fraction should be the better choice for treatment of early breast cancer patients [3].

\section{Review}

Since the 1970s, several trials have employed hypofractionated schedules, with doses[2.2 Gy/fraction and lower total doses [4,5]. Now. For women with invasive breast cancer receiving Whole breast irradiation ( WBI ) with or without inclusion of the low axilla, the preferred dose-fractionation scheme is hypofractionated WBI to a dose of 4000 cGy in 15 fractions or 4250 cGy in 16 fractions according to American society of Radiation Oncology (ASTRO) [6] while extreme hypofractionation became an option according to the very recent UK FAST- Forward trial [7].

Interest in hypofractionation applied to primary breast cancer was sparked by the Canadian radiation biologist Bruce Douglas. On the final page of his 11-page manuscript of the of the still-new linear quadratic model was a single reference to hypofractionation; "For breast cancer, however, the $\beta / \alpha$ value that can be calculated from published data is about 0.26 Since the reciprocal of 0.26 is an $\alpha / \beta$-value of 3.8 Gy $[8,9]$. The data to which Douglas referred were published by Lionel Cohen in the British Journal of Radiology in 1952 [10]. His manuscript combined an analysis of patients treated at the Radiotherapy Department, Johannesburg, with a review of earlier manuscripts describing tumor control in $>1000$ locally advanced or recurrent breast cancer patients irradiated with a range of fractionation regimens (Figure 1). Douglas does not describe how he reanalysed Cohen's data, but he will have controlled for time-related effects by estimating median tumor control doses for subgroups of patients treated to different total doses in daily fractions over similar time periods.

\section{Radiobiological Background}

Conventional fractionation (CF) for half a century has been justified on the basis that 2.0 Gy fractions spare dose limiting late- 


\section{Cancer Therapy \& Oncology International Journal}

responding normal tissues to a greater degree than cancerous tissues [9]. In the landmark study by Owen et al. In 1986-98, randomly assigned 1410 women with invasive breast cancer (tumor stage 1-3 with a maximum of one positive node and no metastasis) who had had local tumour excision of early stage breast cancer to receive 50 Gy radiotherapy given in 25 fractions, 39 Gy given in 13 fractions, or 42.9 Gy given in 13 fractions, all given over 5 weeks. The primary endpoint was late change in breast appearance. ipsilateral tumor relapse, one of the secondary endpoints. It was confirmed that the sensitivity of breast cancer to dose per fraction was estimated to be $4 \cdot 0$ Gy $(95 \%$ CI 1·0-7·8), similar to that estimated for the late adverse effects in healthy tissue from breast radiotherapy. Interpretation of this is that Breast cancer tissue is probably just as sensitive to fraction size as dose-limiting healthy tissues. This study suggested that "If this finding is confirmed, radiotherapy schedules can be greatly simplified by the delivery of fewer, larger fractions without compromising effectiveness or safety, and possibly improving both" [11].

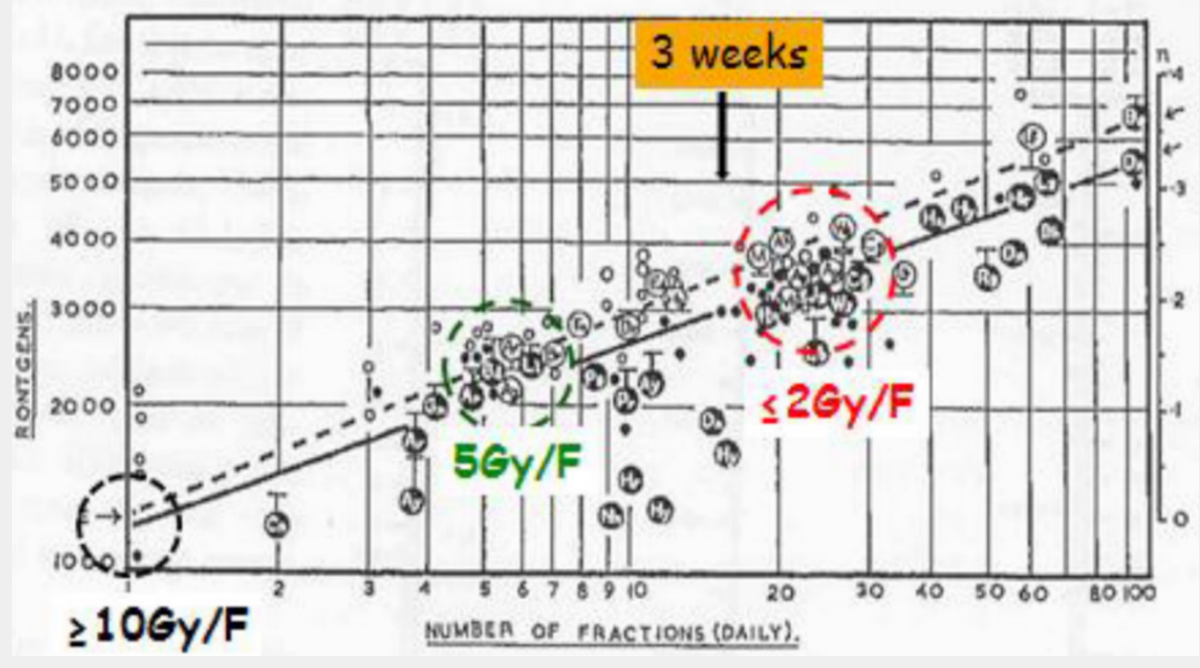

Figure 1: Dose-effect for control of inoperable or recurrent breast cancer. adapted from Cohen[11] . Vertical axis is total dose expressed in Roentgens; Vertical position indicates median tumour dose prescribed. Horizontal axis indicates number of daily fractions given 5-times weekly. Large circles represent published series of minimum 30 patients; open large circles describe control in which $\geq 50 \%$ tumor control; closed large circles describe control in which $<50 \%$ tumour control. Small circles/dots represent individual patients treated by Cohen; open symbol indicates local control; closed symbol indicates local failure. Dashed line represents the median tumor control dose for 61 of Cohen's patients. Solid line represents median tumour control doses for previously published series.

Breast cancer is more sensitive to fraction size than many other common cancers. Early indications that breast cancer responds more strongly to fraction size than many other common cancers and there is now reliable Level I evidence that this is the case. Four randomized trials testing fraction sizes in the range 2.7-3.3 Gy have reported 10-year follow up in almost 8000 patients, providing estimates of $\alpha / \beta$ in the range of 3 Gy. The implication is that there is no superiority in terms of safety or effectiveness of $2.0 \mathrm{~Gy}$ fractions in patients with breast cancer. Hypofractionated (HF) 15 or 16 fraction schedules have been replacing the conventional 25 -fraction regimen as a standard of care for adjuvant therapy in an increasing number of countries. The lower limits of hypofractionation are currently reaching a 5 -fraction schedule of local-regional radiotherapy delivered in 1 week $[7,9]$. A number of concerns are still not answered.

The conventional fractionation (1.8-2 Gy per fraction) has been used as the standard over the last decades, with a total dose of 50-50.4 Gy over 25-28 sections, distributed over 5 consecutive weeks [12]. The use of small fractions $(\leq 2 \cdot 0 \mathrm{~Gy})$ in curative radiotherapy is strongly based on reliable data for squamous carcinomas of the bronchus, cervix uteri, and head and neck ,which show that these tumors are much less sensitive than lateresponding healthy tissues to the size of individual fractions. If these cancers are treated with fractions of greater than $2 \cdot 0 \mathrm{~Gy}$, the ratio of cured local tumors to late complications is lower than if smaller fractions are given during the same overall treatment time [13].

Hypofractionated radiotherapy is an RT technique in which the total dose is administered over a shorter time range with fractionated doses that are higher than the conventional ones. The scientific evidence for its use is established by prospective and randomized studies, including a considerable number of patients submitted to the conventional treatment for breast cancer, with comparable safety, effectiveness, local control, and survival observed on conventional treatment $[6,14,15]$. 
For late-responding tissues $\alpha / \beta$ generally ranges from 0.5 to 6 Gy. The fractionation response of well-oxygenated carcinomas of head and neck, and lung, are thought to be similar to that of early responding normal tissues. However, there is clear evidence that some human tumor types like prostate, breast, melanoma, and sarcomas exhibit low $\alpha / \beta$ values, maybe with $\alpha / \beta$ even lower than for some late-responding normal tissues. late-reacting normal tissues being more sensitive to fraction size than early reacting normal tissues and most cancers (Figure 2) [16].

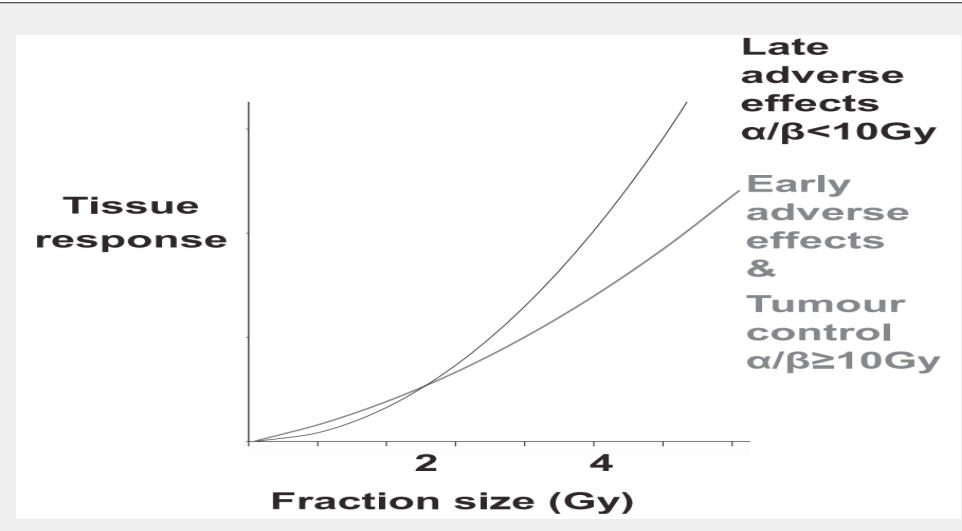

Figure 2: Schema illustrating the traditional model of fractionation sensitivity in normal and malignant tissues, late-reacting normal tissues being more sensitive to fraction size than early-reacting normal tissues and most cancers [17].

From (Figure 3) It can be seen that Late-responding tissues are more sensitive to a change in dose per fraction and this could be interpreted as reflecting a greater curvature of an underlying survival curve for putative target cells. When dose per fraction is increased above a reference level of $2 \mathrm{~Gy}$, the is effective dose falls more rapidly for the late-responding tissues than for the early responses. Similarly, when dose per fraction is reduced below $2 \mathrm{~Gy}$, the is effective dose increases more rapidly in the lateresponding tissues [17].

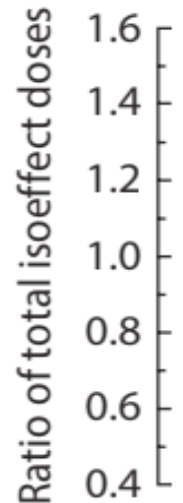

(a)

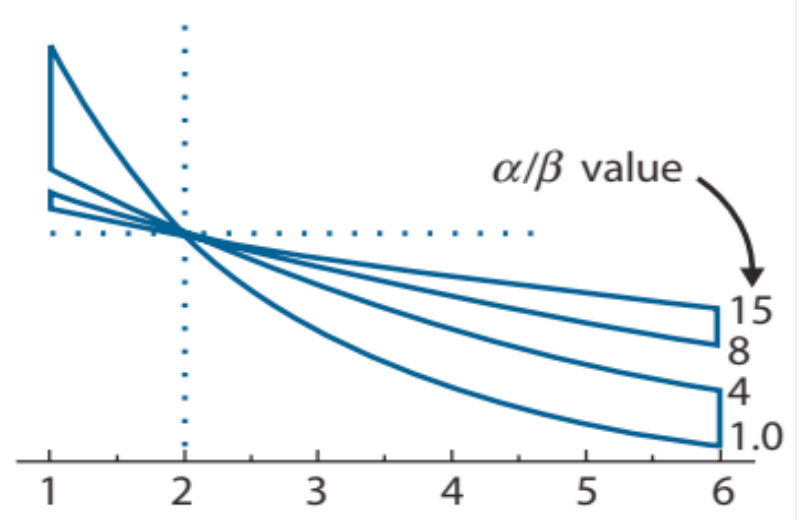

Dose per fraction (Gy)

Figure 3: Theoretical isoeffect curves based on the LQ model for various $\alpha / \beta$ values [18].

The use of fewer fractions of more than $2 \cdot 0$ Gy (hypofractionation) in primary breast cancer is based on data suggesting that breast adenocarcinoma is more sensitive to fraction size than squamous carcinomas, and therefore could have similar fractionation sensitivity to the dose-limiting healthy tissues, including skin, subcutaneous tissues, muscle, and ribs
$[8,10,18,19]$. The response to fraction size is typically non-linear and well fitted by a linear-quadratic function in which clinical response is proportional to $\alpha \mathrm{D}+\beta \mathrm{D} 2$, where $\alpha$ and $\beta$ are If breast cancer coefficients characteristic of the tissue under consideration and D is the fraction size [13]. 
Assuming a typical a/b value of 3.0 Gy for late normal tissue responses, a 15-fraction regimen reproducing the effects of 25 fractions of 2.0 Gy requires a reduction in total dose from $50 \mathrm{~Gy}$ to $42.8 \mathrm{~Gy}$ in fractions of $2.85 \mathrm{~Gy}$ [20]. The linear-quadratic model predicts that the Ellis formula estimate of $45 \mathrm{~Gy}$ in 15 fractions is equivalent to 54 Gy in 2.0 Gy fractions, or to 56.3 Gy in the case of tissues like the brachial plexus with an assumed a/b value of $2.0 \mathrm{~Gy}$. Thus, using the Ellis formula for estimating biologically is effective doses for late effects leads to an overdose of the tissues where these effects are dose-limiting [1]. If breast cancer is generally as sensitive to fraction size as are the late-reacting healthy tissues of the breast, muscle, and underlying ribcage (ie, an $\alpha / \beta$ value of 3-5 Gy compared with $\geq 10$ Gy for squamous carcinomas), larger fraction sizes will be more effective than previously thought [11].

\section{Clinical Evidence}

A randomized clinical trial [1] was therefore begun in 1986 to test the hypothesis that fewer, larger fractions are at least as effective as standard fractions of $2 \cdot 0$ Gy and offer convenience and reduced cost for patients and health services. The study was planned with late effects of healthy tissue as the primary endpoint and tumour recurrence and palpable fibrosis as the secondary endpoints. The trial was controlled for overall treatment time of radiotherapy, and generated reliable estimates of $\alpha / \beta$ of 3.6 Gy (95\% CI 1.8-5.4) for late change in breast appearance (assessed by photographs taken before and after treatment) and of $3 \cdot 1 \mathrm{~Gy}$ $(1 \cdot 8-4 \cdot 4)$ for moderate or striking breast induration (grade 2-3 on a clinical examination scale of 4 ).

The early results informed the design of the UK National Cancer Research Institute (NCRI) Standardization of Radiotherapy (START) trial [21], launched in January 1999, which was planned to have sufficient statistical power to assess tumor control as the primary endpoint. In the study of Owen et al. that found that the sensitivity of breast cancer to dose per fraction was estimated to be $4 \cdot 0$ Gy $(95 \%$ CI $1 \cdot 0-7 \cdot 8)$, similar to that estimated for the late adverse effects in healthy tissue from breast radiotherapy. Interpretation for that Breast cancer tissue is probably just as sensitive to fraction size as dose-limiting healthy tissues. If this finding is confirmed, radiotherapy schedules can be greatly simplified by the delivery of fewer, larger fractions without compromising effectiveness or safety, and possibly improving both [11].

Zhi-Rui Zhou in a meta-analysis conducted in 2015 concluded that HF Radiation Therapy with 2.5-3.0 Gy per fraction should be the better choice for treatment of early breast cancer patients [3]. In conclusion, on the basis of level I evidence from four clinical trials, there appears to be no reason to avoid modest hypofractionation for the adjuvant treatment of women requiring whole breast or postmastectomy chest wall radiotherapy in any identifiable subset of subclinical breast cancers. However, there are some residual concerns [1].

Extreme hypofractionation: The UK FAST trial tested two total doses (30 and $28.5 \mathrm{~Gy}$ ) of a 5-fraction regimen (3.0 \& 5.7 Gy per fraction) delivering one fraction per week against 50 Gy in 2.0 Gy fractions to whole breast in 915 females $\geq 50$ years following complete microscopic local resection of pT1-2pN0M0 disease [22]. Median 3-year analyses of adverse effects (primary end point) were consistent with $\alpha / \beta$ of $3 \mathrm{~Gy}$, as reported by the START trials, although the point estimate was slightly lower at 2.6 Gy [95\% CI (1.4-3.7)]. If a point estimate of 2.6 Gy is assumed, it may reflect the much lower rate of moist desquamation $(11 \%$ after $50 \mathrm{~Gy}$ vs $2 \%$ after $28.5 \mathrm{~Gy}$ ) and reduced risk of consequential late effects, particularly telangiectasia in the inframammary fold. Consequential late effects share the same high $\alpha / \beta$-value as the epidermal depletion that causes them and if present will tend to increase the estimate of $\alpha / \beta$-value. At last reporting (median 3 years), only two local relapses had been recorded [22] (Table 1).

Table 1: Patient and treatment characteristics in four randomized trials testing hypofractionated radiotherapy after surgery for early breast cancer $[8,10]$.

\begin{tabular}{|c|c|c|c|c|c|}
\hline & START-P & START-A & START-B & Ontario & Fast-forward \\
\hline Years accrual & $1986-1998$ & $1998-2002$ & $1999-2001$ & $1993-1996$ & $2011-2014$ \\
\hline Total number of patients & 1410 & 2236 & 2215 & 4096 \\
\hline Standard arm (Gy/fr/weeks) & $50 / 25 / 5$ & $50 / 25 / 5$ & $50 / 25 / 5$ & $50 / 25 / 5$ & $40 / 15 / 3$ \\
\hline Test arm A (Gy/fr/weeks) & $42.9 / 13 / 5$ & $41.6 / 13 / 5$ & $40.0 / 15 / 5$ & $42.5 / 16 / 3.1 \mathrm{n} / \mathrm{a}$ & $27-05-2001$ \\
\hline Test arm B (Gy/fr/weeks) & $39 / 13 / 5$ & $39 / 13 / 5$ & $\mathrm{n} / \mathrm{a}$ & $\mathrm{n} / \mathrm{a}$ & $26-05-2001$ \\
\hline Mean age (years) & 54.5 & 57.2 & 57.4 & Not reported & 61 \\
\hline Node+ (\%) & 32.7 & 28.8 & 22.8 & 0 & 18.7 \\
\hline Mastectomy (\%) & 0 & 15 & 8 & 0 & 19.3 \\
\hline
\end{tabular}




\section{Cancer Therapy \& Oncology International Journal}

\begin{tabular}{|c|c|c|c|c|c|}
\hline Tumour size $\geq \mathrm{T} 2(\%)$ & $42.5^{\mathrm{a}}$ & $48.6^{\mathrm{b}}$ & $35.9^{\mathrm{b}}$ & $20.0^{\mathrm{b}}$ & 0 \\
\hline Boost (\%) & 74.5 & 60.6 & 42.6 & 11.3 & 50.8 \\
\hline Chemotherapy (\%) & 13.9 & 35.5 & 22.2 & 0 & 0 \\
\hline Regional Radiotherapy (\%) & 20.6 & 14.2 & 7.3 & 0 \\
\hline
\end{tabular}

${ }^{a}$ Clinical

${ }^{\mathrm{b}}$ Pathological

Very recently the UK (FAST-Forward) trial was published [7] which is a multicenter, phase 3 , randomized, non-inferiority trial done with Patients with invasive carcinoma of the breast (pT1-3, pN0-1, M0) after breast conservation surgery or mastectomy allocated patients to either 40 Gy in 15 fractions/3 weeks, 27 Gy in five fractions / 1 week, or 26 Gy in five fractions / 1 week) to the whole breast or chest wall. Study showed higher normal tissue effect risk for 27 Gy versus 40 Gy but not for 26 Gy versus 40 Gy. Interpretation and concluded that 26 Gy in five fractions over 1 week is non-inferior to the standard of $40 \mathrm{~Gy}$ in 15 fractions over 3 weeks for local tumour control, and is as safe in terms of normal tissue effects up to 5 years for patients prescribed adjuvant local radiotherapy after primary surgery for early-stage breast cancer

Arguments against routine adoption of 15- or 16-fraction regimens given Level I evidence of safety and effectiveness are often expressed in terms of underrepresented patient subgroups beside rapid changes in patient management. Subgroups for endocrine and biological agents that target cancer-specific mechanisms.

ASTRO recommendations for hypofractionated radiation selection of patients has changed within a decade in the 2011 guidelines [23] it was confined to patients with early stage (T2,T2) node negative above 50 years with no adjuvant chemotherapy given and with dose hemogenity of $\pm 7 \%$ along central axis while it was extended to include Any age ,any stage with or without nodal fields and with minimizing area receiving less than $105 \%$ [6].

Important Highlights from the ASTRO guidelines 2018 [6]:

i. Conventional fractionation (CF-WBI) may be preferred over hypofractionated whole breast irradiation (HFWBI) when treating primary breast cancers with rare histologies that are most commonly treated with CF when arising in other parts of the body with $93 \%$ agreement and low level of evidence.

ii. The decision to offer HF-WBI should be independent of breast size (including central axis separation) provided that dosehomogeneity goals, can be achieved with moderate evidence and $100 \%$ agreement.

iii. In patients with breast augmentation, either HF-WBI or CF-WBI may be used with low evidence and $85 \%$ agreement. iv. In patients with collagen vascular disease, if the patient and her physician opt for WBI, then either HF-WBI or CF-WBI may be used with $85 \%$ agreement and low evidence.

\section{Special Concerns}

\section{Age}

Start ( A, and B ) and Canadian studies included, respectively, $23 \%, 21 \%$, and $25 \%$ of women under 50 years old. The local control was similar among different ages $[9,15,24]$. There was NO agreement regarding the safety of indication of hypofractionated RT of exclusively the breast for patients with age under 40 years [12].

\section{Breast Size}

The Canadian study limited the inclusion of breasts with latero-lateral diameter above $25 \mathrm{~cm}^{2}$ [15]. He Start studies (A, and B) did not limit breast size, and classified them into small, medium, and large. There was no toxicity difference amongst different breast sizes. The restriction should be made according to dosimetric parameters [12,14].

\section{Chemotherapy}

None of the clinical trials that assessed hypofractionated RT included neoadjuvant CT in their respective treatment protocols; however, the indication in this scenario has substantially increased over the last few years. Adjuvant Chemotherapy was used in $13.9 \%, 35.5 \%, 22.2 \%$, and $11 \%$ in the Start-P15 adjuvant CT. There was no difference in local control in the Start (P, A, and B ) and Canadian $[9,15,24,25]$ studies in the subgroup of adjuvant CT, regardless of the RT model used (hypofractionated RT or conventional RT).There was no difference amongst the study groups, upon evaluating cosmesis and regular tissue toxicity, regardless of the use of adjuvant CT.

The use of hypofractionated RT for breast cancer has been increasing considerably over the years. A study conducted by the US National Cancer Database showed an increase in hypofractionated RT indication for patients who received CT, with an absolute increase of $13.6 \%$ over the last decade (from $4.6 \%$ to $18.2 \%$ ) and this was confirmed by Diwanji et al. that the use of HF whole breast radiation therapy in patients receiving chemotherapy has increased fourfold (absolute $=13.6 \%$ ) over a decade from 2004 to $2013[26,27]$. In current clinical practice, the exposure to CT, 
both adjuvants as neoadjuvant, prior to surgery did not alter the toxicity patterns for hypofractionated RT [12]. The is no data in the literature that addresses the oncologic safety of hypofractionated RT concurrent with CT since the main clinical trials available did not use that combination [26]. Hypofractionated RT, exclusively concurrent with anti-HER2 drugs, is considered to be safe [12].

\section{Mastectomy}

The hypofractionation trials were conducted before the current era of oncoplastic surgery, but there are no identifiable reasons to avoid hypofractionation in this subgroup either. As emphasised above, even assuming $\alpha / \beta$ of $1.5,40$ Gy in 15 fractions is expected to generate milder late adverse effects than $50 \mathrm{~Gy}$ in 25 fractions [9].

\section{Regional Nodal Irradiation}

There were no randomized clinical trials that included the axilla in RT volumes. Despite some studies suggesting the model were equivalent regarding acute and late toxicities, most panel members did not consider hypofractionated radiotherapy to be appropriate in this context due to a lack of safety for recommendation to this day [12]. The Start (P, A, and B ) used hypofractionated RT in the SCF, respectively, in $20 \%, 14 \%$, and $7 \%$ of a total of 470 patients. The Canadian [15] study did not include patients for lymphatic drainage irradiation $[9,24,25]$. The Cochrane [27] meta-analysis grouped only $10 \%$ of patients who underwent hypofractionated RT in the SCF.

In total, only 470 patients were prescribed lymphatic radiotherapy in the START trials, most commonly to supraclavicular fossa, and extending to upper axillary levels in a small minority. Variable volumes of Level I/II axilla would have been included the tangential beams to the breast, despite Level I/II surgical dissection in $>90 \%$ patients [9]. There was no suggestion of enhanced shoulder stiffness or arm oedema associated with hypofractionation in the 10-year START assessments, but these included all patients regardless of lymphatic radiotherapy [14].

A Chinese study [28] randomized 811 patients with highrisk breast cancer, stage II, for conventional or hypofractionated RT in the SCF and did not observe any difference in locoregional recurrence, distant metastasis, disease free survival, and global survival. Locoregional recurrence was also similar in meta-analysis [3] [relative risk [RR] $=1.03 ; 95 \% \mathrm{CI}(0.87 ; 1.23), \mathrm{P}=0.72$ ], and in the Start [9] studies $(0.5 \%$ vs $0.3 \%$; $=0.71)$. The risks of pulmonary toxicity, rib fracture, plexopathy, and upper limb lymphedema were similar between the conventional and hypofractionated RT models $[3,9]$. There was NO agreement regarding the safety of indication of hypofractionated RT exclusively of the breast in lymphatic drainage of the supraclavicular fossa (SCF) and axilla.

Internal Mammary chain: The randomized studies did not include the internal mammary chain in the RT volumes. Despite some studies suggesting equivalent levels of acute and late toxicity, it is not possible to exclude the possibility of increased pulmonary, costal arch, and heart toxicity with hypofractionated radiotherapy due to lack of scientific evidence [3].

\section{Boost}

In the Start studies (Start-P, Start-A, and Start-B ) and the Cochrane meta-analysis $[9,24,25,27]$, a boost was used in $75 \%$, $60 \%, 43 \%$, and $44 \%$ of patients, respectively. In MD Anderson [29] and a Chinese study [3], all patients had a boost after the whole breast hypofractionated RT. No increased toxicity was observed with the addition of a boost to the hypofractionated models when compared to conventional therapy $[3,27]$. The models used were: $3 \times 3$ Gy, 4x2,5 Gy, 3x2,67 Gy and 5x2 Gy. The UK IMPORT HIGH trial $(\mathrm{N}=2,658)$ tests the hypothesis that concomitant boost is as safe and non- inferior to standard sequential boost techniques .

\section{T3 Stage}

T3 stage was included in the Start studies (Start-P, Start-A, and Start-B), with tumors equal or larger than T2 representing $42.5 \%, 48.6 \%$, and $35.9 \%$, respectively. There is no analysis of the results; however, the randomized controlled trials considered that the size of the resected tumor, on its own, should not be an exclusion factor for hypofractionated radiotherapy [9,24,25].

\section{Breast Reconstruction}

Despite Start studies (A and B ) [14] not having as the assessment of post-mastectomy hypofractionated RT as the initial objective, this group represented $8 \%$ (513 patients) of the sample [14]. There was no statistical power for a recommendation. However, locoregional recurrence happened in $6.8 \%$ of these patients. The toxicity was not different for patients who underwent mastectomy and hypofractionated RT. Radiobiological ratios of similar remaining-tissue sensibility, regardless of the surgical technique, and the potential reduction of late events from breast $\alpha / \beta$ encourage the use of hypofractionated models [1].

The Startstudies (A and B) excluded post-mastectomy patients with immediate reconstruction, and there are no other studies that can be used as a reference for the procedure There was NO agreement as to the safety of the indication of post-mastectomy hypofractionated RT after immediate breast reconstruction WITH autologous tissue. The Start studies (A and B ) excluded postmastectomy patients with immediate reconstruction and, to the present day, there are no other studies with results that can be used as a reference for the procedure [12,13].

\section{Ductal carcinoma in situ (DCIC)}

Hypofractionation use was similar in patients with DCIS and those with invasive disease [30].

\section{Critical Organs}

Lung: The sensitivity of lung tissue to larger fractions is a concern, but lung doses delivered by tangential fields exceed tolerance in whatever fractionation schedule is used. It is unusual 


\section{Cancer Therapy \& Oncology International Journal}

for patients to develop clinically significant pneumonitis or fibrosis following radiotherapy confined to the whole breast [31,32]. The Heart: Since there appears to be no safe lower dose limit, however fractionated the priority is to protect the heart from exposure regardless of radiation schedule [33,34].

After irradiation of the axilla and/or supraclavicular fossa, there were no cases of brachial plexopathy recorded in 82 patients given 40 Gy in 15 fractions in the START B trial at a median followup of 6.0 years (interquartile range [IQR], 5.0-6.2 years) [35]. The regimen is equivalent to 47 Gy in 2.0-Gy fractions if the a/b value for brachial plexus is 2.0 Gy or to 49 Gy in 2.0-Gy fractions if a/b = $1.0 \mathrm{~Gy}$. If radiotherapy centers are confident that their technique is safe when prescribing 50 Gy in 25 fractions, there will be no excess risk after 40 Gy in 15 fractions by using the same treatment position, field arrangement, dosimetry, and reference point [1].

\section{"Triple-trouble"}

"Double trouble" was the term coined by Withers [36] to illustrate the significance of a hot spot in a dose plan that receives not only a higher total dose but also a higher dose per fraction. However, due to the mathematical form of the linear-quadratic dose-effect relationship, hot spots will be penalized more severely in a hypofractionated treatment, a phenomenon called triple-trouble [37]. One way to look at this is by noticing that the steepness of the dose-response curve increases with increasing dose per fraction and that this will tighten the required dose uniformity [38].

For distributions falling within International Commission on Radiation Units and Measurements recommendations (95\%$107 \%$ of reference isodose), triple-trouble has no clinically relevant impact: the main effect is double-trouble, already present in a 2-Gy-per-fraction schedule. Even for hot spots of $>110 \%$, there is a marked volume response for late effects such as breast shrinkage and induration. In other words, the clinical consequences of high doses to small volumes are much less than high doses delivered to large volumes [1]. In conclusion : Current evidence suggesting non inferiority of hypofractionation radiation therapy for the adjuvant therapy of breast cancer with better cosmetic and late effect. Extreme hypofractionation is very recently also proved non inferiority for early stage adjuvant therapy. Data heterogeneity of patient populations is still a challenge to adobt a universally accepted protocol.

\section{References}

1. Darby S, McGale P, Correa C, Taylor C, Arriagada R, et al. (2011) Effect of radiotherapy after breast-conserving surgery on 10-year recurrence and 15-year breast cancer death: meta-analysis of individual patient data for 10801 women in 17 randomised trials. The Lancet 378(9804): $1707-1716$

2. Yarnold J, Bentzen SM, Coles C, Haviland J (2011) Hypofractionated whole-breast radiotherapy for women with early breast cancer: myths and realities. Int J Radiat Oncol Biol Phys79(1): 1-9.
3. Zhou ZR, Mei X, Chen XX, Yang ZZ, Hou J et al. (2015) Systematic review and meta-analysis comparing hypofractionated with conventional fraction radiotherapy in treatment of early breast cancer. Surgical oncology 24(3): 200-211.

4. Clark RM, Whelan T, Levine M, Roberts R, Willan A, McCulloch P, et al. (1996) Randomized clinical trial of breast irradiation following lumpectomy and axillary dissection for node-negative breast cancer: an update. J Natl Cancer Inst 88(22): 1659-1664.

5. Forrest AP, Stewart HJ, Everington D, Prescott RJ, McArdle CS, et al (1996) Randomised controlled trial of conservation therapy for breast cancer: 6-year analysis of the Scottish trial. The Lancet 348(9029): 708-713.

6. Smith BD, Bellon JR, Blitzblau R, Freedman G, Haffty B, et al. (2018) Radiation therapy for the whole breast: Executive summary of an American Society for Radiation Oncology (ASTRO) evidence-based guideline. Practical radiation oncology 8(3): 145-152.

7. Adrian Murray Brunt, Joanne S Haviland, Duncan A Wheatley, Mark A Sydenham, Abdulla Alhasso,

et al. (2020) Hypofractionated breast radiotherapy for 1 week versus 3 weeks (FAST-Forward): 5-year efficacy and late normal tissue effects results from a multicentre, non-inferiority, randomised, phase 3 trial. The Lancet.

8. Douglas B, J Castro (1984) Novel fractionation schemes and high linear energy transfer. Progress in experimental tumor research 28: 152-165.

9. Yarnold J (2018) Changes in radiotherapy fractionation-breast cancer. Br J Radiol 92(1093): 20170849.

10. Cohen L (1952) The Dose-time Relationship: Theoretical Considerations. Br J Radiol 25(300): 636-642.

11. Owen JR, Ashton A, Bliss JM, Homewood J, Harper C, et al. (2006) Effect of radiotherapy fraction size on tumour control in patients with earlystage breast cancer after local tumour excision: long-term results of a randomised trial. The lancet oncology 7(6): 467-471.

12. Nilceana Maya Aires Freitas, Arthur Accioly Rosa, Gustavo Nader Marta, Samir Abdalla Hanna, Rodrigo de Morais Hanriot, et al. (2018) Recommendations for hypofractionated whole-breast irradiation. Revista da Associação Médica Brasileira 64(9): 770-777.

13. SM Bentzen, MC Joiner (2002) The linear-quadratic model in clinical practice. Basic clinical radiobiology, pp. 134-146.

14. Haviland JS, Owen JR, Dewar JA, Agrawal RK, Barrett J, et al. (2013) The UK Standardisation of Breast Radiotherapy (START) trials of radiotherapy hypofractionation for treatment of early breast cancer: 10-year follow-up results of two randomised controlled trials. The lancet oncology 14(11): 1086-1094.

15. Whelan TJ, Jean-Philippe Pignol, Mark N Levine, Jim A Julian, Robert MacKenzie, et al. (2010) Long-term results of hypofractionated radiation therapy for breast cancer. New England Journal of Medicine 362(6): 513-520.

16. Somaiah NK, Rothkamm, J Yarnold (2015) Where do we look for markers of radiotherapy fraction size sensitivity? Clinical Oncology 27(10): 570-578.

17. Joiner MC, A Van der Kogel (2016) Basic clinical radiobiology. CRC press, Vol. 1.

18. Thames HD, Hendry JH, Moore JV, Ang KK, Travis EL, et al. (1989) The high steepness of dose-response curves for late-responding normal tissues. Radiotherapy and Oncology 15(1): 49-53.

19. Thames HD, Bentzen SM, Turesson I, Overgaard M, Van den Bogaert W, et al. (1990) Time-dose factors in radiotherapy: a review of the human data. Radiotherapy and Oncology 19(3): 219-235. 


\section{Cancer Therapy \& Oncology International Journal}

20. Jones B, Dale RG, Deehan C, Hopkins KI, Morgan DA et al. (2001) The role of biologically effective dose (BED) in clinical oncology. Clinical oncology 13(2): 71-81.

21. Hopwood P, Haviland J, Mills J, Sumo G, M Bliss J, et al. (2007) The impact of age and clinical factors on quality of life in early breast cancer: an analysis of 2208 women recruited to the UK START Trial (Standardisation of Breast Radiotherapy Trial). The Breast 16(3): 241 251.

22. Agrawal R, Alhasso A, Barrett-Lee PJ, Bliss JM, Bliss P, et al. (2011) First results of the randomised UK FAST Trial of radiotherapy hypofractionation for treatment of early breast cancer (CRUKE/04/015): The FAST Trialists group. Radiother Oncol 100(1) 93-100.

23. Smith BD, Bentzen SM, Correa CR, Hahn CA, Hardenbergh PH, et al. (2010) Fractionation for whole breast irradiation: an American Society for Radiation Oncology (ASTRO) evidence-based guideline Int J Radiat Oncol Biol Phys 81(1): 59-68.

24. Valle LF, Agarwal S, Bickel KE, Herchek HA, Nalepinski DC, et al. (2017) Hypofractionated whole breast radiotherapy in breast conservation for early-stage breast cancer: a systematic review and meta-analysis of randomized trials. Breast Cancer Res Treat 162(3): 409-417.

25. Yarnold J, Ashton A, Bliss J, Homewood J, Harper C, et al. (2005) Fractionation sensitivity and dose response of late adverse effects in the breast after radiotherapy for early breast cancer: long-term results of a randomised trial. Radiother Oncol 75(1): 9-17

26. Diwanji TP, Molitoris JK, Chhabra AM, Snider JW, Bentzen SM et al (2017) Utilization of hypofractionated whole-breast radiation therapy in patients receiving chemotherapy: a National Cancer Database analysis. Breast Cancer Res Treat 165(2): 445-453.

27. Hickey BE, James ML, Lehman M, Hider PN, Jeffery M, et al. (2016) Fraction size in radiation therapy for breast conservation in early breast cancer. Cochrane Database Syst Rev 7: CD003860.

28. Sun G, S L Wang, YW Song, Z H Yu, X F Liu, et al. (2017) Hypofractionated radiation therapy after mastectomy for the treatment of high-risk breast cancer: 5-year follow-up result of a randomized trial. Int J Radiat Oncol 99(2): S3-S4.
29. Shaitelman SF, Schlembach PI, Arzu I, Ballo M, Bloom ES et al. (2015) Acute and short-term toxic effects of conventionally fractionated vs hypofractionated whole-breast irradiation: a randomized clinical trial. JAMA oncology 1(7): 931-941.

30. Jagsi R, Falchook AD, Hendrix LH, Curry H, Chen RC et al. (2014) Adoption of hypofractionated radiation therapy for breast cancer after publication of randomized trials. Int J Radiat Oncol Biol Phys 90(5): 1001-1009.

31. Rancati T, Wennberg B, Lind P, Svane G, Gagliardi G, et al. (2007) Early clinical and radiological pulmonary complications following breast cancer radiation therapy: NTCP fit with four different models. Radiother Oncol 82(3): 308-316.

32. Lind PA, Wennberg B, Gagliardi G, Rosfors S, Blom-Goldman U, et al (2006) ROC curves and evaluation of radiation-induced pulmonary toxicity in breast cancer. Int J Radiat Oncol Biol Phys 64(3): 765-770.

33. McGale P, Darby SC (2005) Low doses of ionizing radiation and circulatory diseases: a systematic review of the published epidemiological evidence. Radiat Res 163(3): 247-257.

34. Taylor CW, Nisbet A, McGale P, Darby SC, et al. (2007) Cardiac exposures in breast cancer radiotherapy: 1950s-1990s. Int J Radiat Oncol Biol Phys 69(5): 1484-1495.

35. Bentzen SM, Yarnold JR (2010) Reports of unexpected late side effects of accelerated partial breast irradiation-radiobiological considerations. Int J Radiat Oncol Biol Phys 77(4): 969-973.

36. Withers H R (1987) Biologic basis of radiation therapy, in Principles and practice of radiation oncology.

37. Jones B, Dale RG, Finst P, Khaksar SJ et al. (2000) Biological equivalent dose assessment of the consequences of hypofractionated radiotherapy. Int J Radiat Oncol Biol Phys 47(5): 1379-1384.

38. Bentzen SM (2005) Steepness of the radiation dose-response curve for dose-per-fraction escalation keeping the number of fractions fixed. Acta Oncologica 44(8): 825-828.

\section{Your next submission with Juniper Publishers will reach you the below assets}

- Quality Editorial service

- Swift Peer Review

- Reprints availability

- E-prints Service

- Manuscript Podcast for convenient understanding

- Global attainment for your research

- Manuscript accessibility in different formats

(Pdf, E-pub, Full Text, Audio)

- Unceasing customer service

Track the below URL for one-step submission https://juniperpublishers.com/online-submission.php 